\title{
New Operation Scenarios for Severely Irradiated Silicon Detectors
}

\author{
Gianluigi Casse', A. Affolder, P.P. Allport, H. Brown, C. Wiglesworth \\ University Of Liverpool, Physics Department \\ O. Lodge Lab., Oxford street, L69 7ZE, Liverpool, UK. \\ E-mail: gcasse@hep.ph.Iiv.ac.uk.
}

Recent charge collection measurements after severe hadron irradiation have proved that n-side readout segmented planar silicon detectors can successfully operate up to the doses anticipated for the innermost layers of the upgraded experiments in the future super LHC (sLHC) at CERN. The charge collected by the irradiated sensors is sufficient to guarantee a signal over noise $(\mathrm{S} / \mathrm{N})$ ratio above 10 even for the pixel layers located at the smallest radial distance from the beam line (less than $4 \mathrm{~cm}$ away). The signal depends on the applied bias and voltages as high as $1000 \mathrm{~V}$ could be required to satisfy the minimum signal height for the most exposed detectors. The radiation at the doses of the pixel layers in the sLHC also cause an important increase of the reverse current The high bias voltages and reverse currents cause significant power dissipation and adequate cooling needs to be applied to limit the current well below the thermal-runaway level.

Besides, both the collected charge and the reverse current change with time after irradiation (annealing). The rate of the changes is a steep function of the temperature: higher temperature cause accelerated annealing. These changes can in fact been exploited to extend the operation time of the silicon sensors. The requirements in term of bias voltage, temperature of the detectors during operation and temperature scenario outside the operation time (to provide the optimised annealing to the sensors) are here presented and discussed.

\footnotetext{
$1 \quad$ Speaker
} 


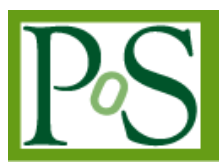

PROCEEDINGS

OF SCIENCE

(c) Copyright owned by the authors) under the terms of the Creative Commons Attribution-NonCommercial-ShareAlike Licence.

http://pos.sissa.it 


\section{Introduction}

The upgrade of the CERN-LHC accelerator (sLHC) will approach the unprecedented luminosity of $\sim 10^{35} \mathrm{~cm}^{-2} \mathrm{~s}^{-1}$ [1]. The tenfold increase of the luminosity with respect to the present machine will lead to the replacement of the vertex and tracker detectors of the experiments to deal with the increased hit density. Higher granularity (to limit the channel hit occupancy) and radiation tolerant detectors will be required. Concerning this last aspect, very encouraging results have been obtained in the last few years that allow for optimism regarding the functionality of the sensors after the hadron doses anticipated for the sLHC [2-6]. Nevertheless the detectors must be operated at high bias voltage and draw a significant reverse current after severe irradiation, adding to the power consumption of the front-end electronics (already considerable due to the fine granularity). An important aspect of the running procedures in the future sLHC experiments will be the choice of the optimal bias voltage and of the temperature of the detectors during operation and shut down times. Data are already available to help designing these procedures for the vertex and tracker detectors at different radii. In order to estimate the performance of the irradiated sensors, a few methods can be followed. In nonirradiated silicon detectors all the charge created by ionisation is collected if the device is biased to its full depletion voltage $\left(V_{F D}\right)$ or above (over-depletion). The ability of over-depleting a detector can be considered as an estimate of its efficiency. This has been used in the design phase of the microstrip sensors of the present tracker systems of the general purpose detectors of the LHC ([7, 8]). This method does not account for the trapping of the signal charge carriers or for the fact that acceptable performance can be achieved also with bias voltage values below $\mathrm{V}_{\mathrm{FD}}$.

A most direct estimate of the performance of the sensors is the measurement of the signal after irradiation. It is generally accepted that good tracking performance can be achieved with a signal over noise ratio of 10 or larger. The noise depends on the detector geometry and electronics chain while the signal degradation is a property of the silicon crystal. The knowledge of the signal height after the relevant irradiation fluence combined with the known properties of the readout electronics allows for an accurate estimation of the performance of a given detector system.

\section{Changes of electrical properties with irradiation}

The hadron radiation damage causes the change of the electrical properties of the silicon detectors. The effective space charge $\left(N_{e f f}\right)$, proportional to $V_{F D}$, increases linearly with fluence after an initial reduction. The reverse current $\left(I_{R}\right)$ increase is also proportional to the radiation fluence. These changes have been parameterised [9] following a large number of measurements with silicon detectors up to fluences of about $1 \times 10^{15} \mathrm{n}_{\mathrm{eq}} \mathrm{cm}^{-2}$, because at more elevated doses the high value of $V_{F D}$ prevents depleting the devices. It should be noticed that $I_{R}$ is an exponential function of the temperature, so low temperatures can be used to efficiently reduce the current. 
The collected charge decreases after irradiation, for a given applied reverse bias, due to two effects: the increase of $V_{F D}$ and the charge trapping at radiation induced defect centres. The increase of $V_{F D}$ causes the reduction of the depleted volume for a given applied voltage below full depletion. In the following, only the signal generated by minimum ionising particles (mip's) is considered, because it is relevant to high energy physics applications. The charge generated by a mip is proportional to the path length of the particle in the sensitive volume of a silicon detector, consequently a reduction of the active volume corresponds to an equivalent diminution of the signal. The charge trapping centres introduced by the irradiation remove charge carriers from the signal current. The density of traps is assumed to increase linearly with fluence and the effective trapping time $\tau_{\mathrm{e}, \mathrm{h}}$ for electrons (e) and holes (h) changes like [10]:

$$
1 / \tau_{e, h}=\beta_{e, h} \phi_{e q}
$$

where $\phi_{e q}$ is the $1 \mathrm{MeV}$ neutron equivalent fluence and $\beta_{e, h}$ is the proportionality constant for electrons and holes. The ratio of the collection time of the signal $\left(t_{S}\right)$ to $\tau_{e, h}$ defines the amount of charge loss to trapping according to:

$$
Q_{\text {signal }}=Q_{0} e^{-\frac{t_{S}}{\tau_{e, h}}}
$$

where $Q_{\text {signal }}$ and $Q_{0}$ are the measured charge and the ionised charge in the active volume, respectively. From Eq. 2, it is clear that shorter $t_{S}$ yield larger signal. The electron signal in irradiated silicon detectors is faster than the signal carried by holes. For this reason, it has been shown that reading out segmented detectors from the $n^{+}$implant (n-in-n or n-in-p geometries) offer a substantial advantage with respect to the more standard diode configuration ( $\mathrm{p}$-in- $\mathrm{n}$ ) after irradiation $[2-6,11,12]$.

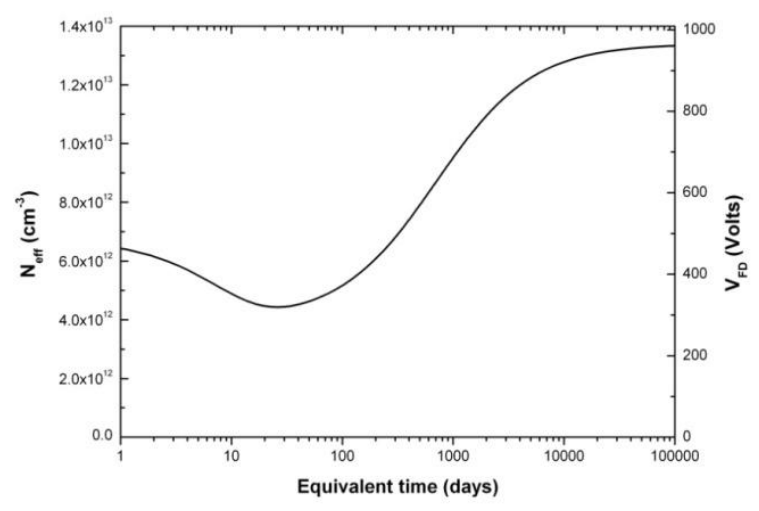

Figure 1: Changes of $N_{\text {eff }}\left(V_{F D}\right)$ with time after irradiation $\left(a t 20^{\circ} \mathrm{C}\right)$ for a p-in-n detector irradiated to $2 \times 10^{14} n_{e q} \mathrm{~cm}^{-2}$.

Notable changes also take place with time after irradiation (annealing). The rate of these changes is an exponential function of temperature, so the annealing can be practically suppressed at $0^{\circ} \mathrm{C}$ or below, or accelerated at more elevated temperatures. The reverse current and the charge trapping probability reduce with time while $V_{F D}$ has more complicated annealing behaviours, with an initial reduction (for about ten days at $\mathrm{RT}=20^{\circ} \mathrm{C}$ ) followed by a sizeable increase over several years at room temperature (RT). Figure 1 shows an example of the 
changes of $V_{F D}\left(N_{e f f}\right)$ over several years at RT, according to the parameterisation in [9] for a ntype bulk silicon sensor irradiated to $2 \times 10^{14} \mathrm{n}_{\mathrm{eq}} \mathrm{cm}^{-2}$.

\section{Experimental results}

\section{Charge collection after irradiation}

Using the above parameterisations it is possible to predict the decrease of the signal as a function of the hadron fluence. In fact the measured signal is degraded by increasing hadron irradiation, as shown in Figure 2. Nonetheless, a signal larger than expected is measured after high fluences. Figure 3 shows the collected charge as a function of the bias voltage $(\mathrm{CC}(\mathrm{V}))$ for proton [11] and neutron [12] irradiated n-side readout detectors after very high doses, compared to the signal estimated using the accepted parameterisation for the charge trapping. The enhanced collected charge can be partly due to the failure of the parameterisation to describe the properties of the silicon detectors above $1 \times 10^{15} \mathrm{n}_{\mathrm{eq}} \mathrm{cm}^{-2}$, but the large discrepancy between experimental data and the estimated maximum signal requires a different explanation.
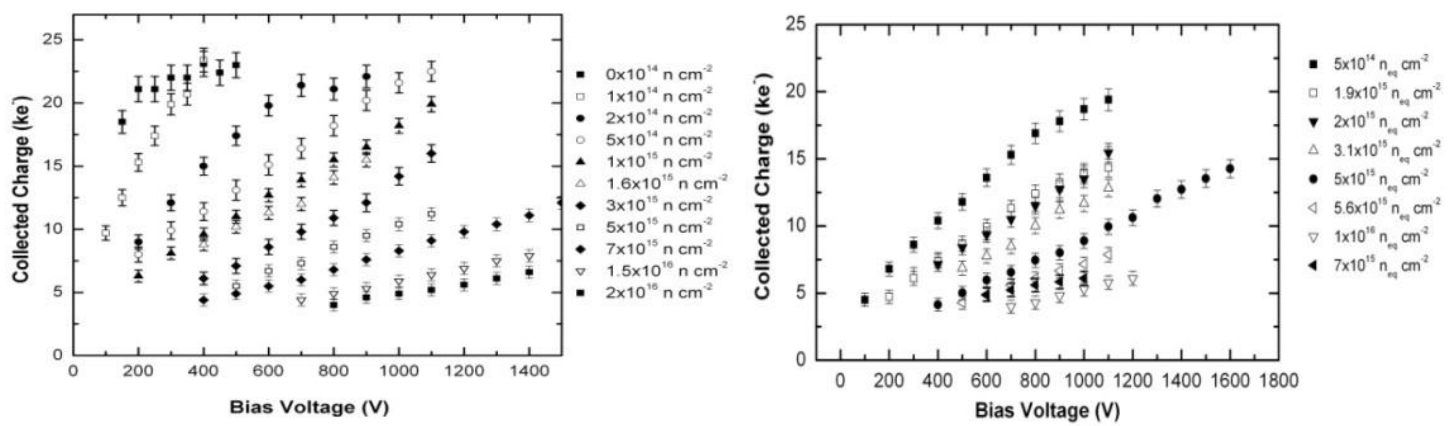

Figure 2: Degradation of the collected charge as a function of the bias voltage $(C C(V))$ for neutron (left) and proton (right) irradiated segmented $300 \mu \mathrm{m}$ thick $n$-in- $p$ silicon sensors.

This remarkable result is believed to be due to a charge multiplication effect at high electric field in irradiated segmented silicon sensors. This interpretation is supported by the results shown in Figure 4, where the CC(V) properties of a thin $(140 \mu \mathrm{m})$ and a standard thickness $(300$ $\mu \mathrm{m})$ silicon microstrip sensors have been measured after proton irradiation to $5 \times 10^{15} \mathrm{n}_{\mathrm{eq}} \mathrm{cm}^{-2}$.
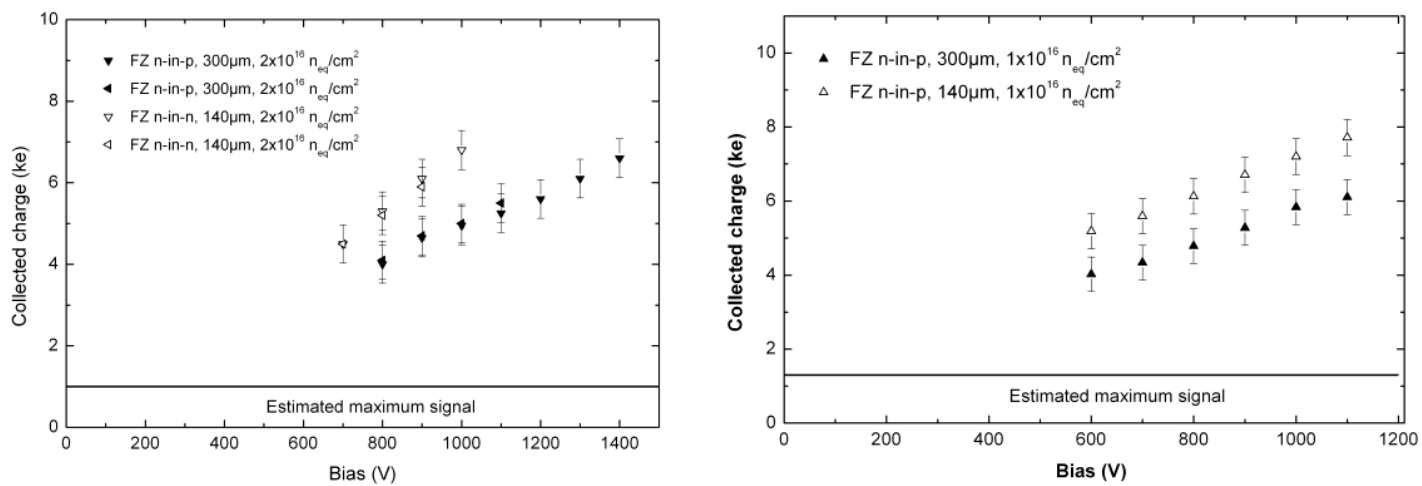

Figure 3: $C C(V)$ of 140 and $300 \mu$ m thick $n$-in-p microstrip detectors irradiated with protons to $1 \times 10^{16}$ $n_{e q} \mathrm{~cm}^{-2}$ (left) and with neutrons to $2 \times 10^{16} n_{e q} \mathrm{~cm}^{-2}$ (right). The figure also shows the expected signal when the charge carrier trapping is considered. 
The charge collected by the thin devices goes up to about $25 \mathrm{ke}$, which is more than twice the charge ionised by a mip in the full volume of a non-irradiated $140 \mu \mathrm{m}$ thick silicon detector, clearly indicating that charge multiplication is taking place.

Another important fact emerging from the measurements is the capability of the irradiated sensors to withstand bias voltages exceeding $1000 \mathrm{~V}$. The measurements here shown have been performed at $-25^{\circ} \mathrm{C}$ to keep the $I_{R}$ under control and avoid thermal runaway. The detectors were operated in stable conditions and continuously for several hours (up to a few days).

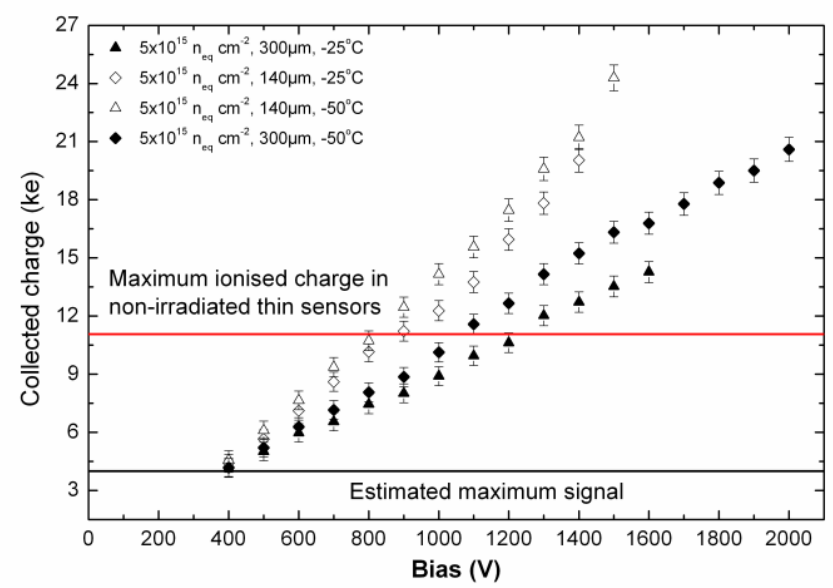

Figure 4: $C C(V)$ of proton irradiated n-in-p microstrip detectors (140 and $300 \mu \mathrm{m}$ thick) after $5 \times 10^{15} n_{\text {eq }}$ $\mathrm{cm}^{-2}$. The figure also shows the expected signal when the charge carrier trapping is considered. The charge collected by the thin device is almost a factor of two higher than the charge deposited by a mip in the full depleted bulk of a non-irradiated sensor. Two set of measurements have been taken with both detectors, at -25 and $-50^{\circ} \mathrm{C}$.
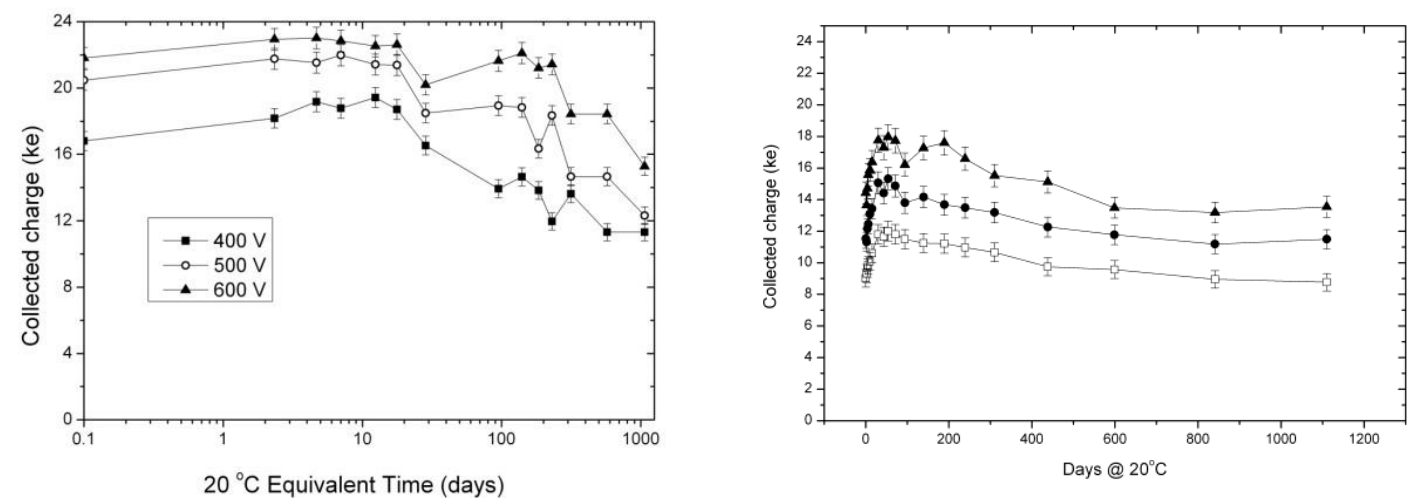

Figure 5: Signal as a function of RT equivalent time of a p-in-n detector irradiated with reactor neutrons to $2 \times 10^{14} n_{\text {eq }} \mathrm{cm}^{-2}$ (left) and a n-in-p detector to $1 \times 10^{I 5} n_{\text {eq }} \mathrm{cm}^{-2}$ (right).

Beside the need for reducing the reverse current, a possible improvement of the charge collection through changes of the carrier mobility and trapping centre occupancies could justify running the irradiated sensors at lower temperatures. Only a rather small difference is though found between the signal measured at $-25^{\circ} \mathrm{C}$ and $-50^{\circ} \mathrm{C}$ (Fig. 4). 


\section{Annealing of the $\mathrm{CC}(\mathrm{V})$}

It could be assumed that the $\mathrm{CC}(\mathrm{V})$ follows closely the annealing of the full depletion voltage, with corrections coming from the annealing of the charge trapping probability that is reducing with time after irradiation [10]. But while these two aspects only depend on the irradiation dose and on the type of the silicon crystal, the annealing of the $\mathrm{CC}(\mathrm{V})$ strongly depends on the readout side. Figure 5 shows the changes of the $\mathrm{CC}(\mathrm{V})$ with time after irradiation for a p-in-n and a n-in-p sensors irradiated to $2 \times 10^{14} \mathrm{n}_{\mathrm{eq}} \mathrm{cm}^{-2}$ and $1 \times 10^{15} \mathrm{n}_{\mathrm{eq}} \mathrm{cm}^{-2}$ with reactor neutrons. To study the annealing behaviour over a few years at room temperature $\left(\mathrm{RT}=20^{\circ} \mathrm{C}\right)$ equivalent time $\left(\mathrm{t}_{\mathrm{eq}}\right)$, the sensors have been taken through controlled heating steps (from 30 minutes to two hours) at 60 or $80{ }^{\circ} \mathrm{C}$, for an acceleration factor of about 540 and 7400 relatively to $\mathrm{RT}$. The $\mathrm{CC}(\mathrm{V})$ of the p-in-n device measured at 500 and 600 volts is essentially stable (the maximum increase is below 5\%) up to 20 days $t_{\text {eq. }}$. Both bias voltages are above the expected value of $V_{F D}$ (Fig. 1) that goes from just under $500 \mathrm{~V}$ to about $320 \mathrm{~V}$ over the same period of time. The signal at $400 \mathrm{~V}$ shows an increase of about $17 \%$, due to $V_{F D}$ going from above to below this bias voltage. Although being fully depleted, a smaller charge is measured at $400 \mathrm{~V}$ with respect to $600 \mathrm{~V}$, which is attributed the more intense charge trapping at the lower bias. After $30 \mathrm{RT}$ equivalent days, the signal at $400 \mathrm{~V}$ and $500 \mathrm{~V}$ keeps degrading and about $30 \%$ of the charge measured at the end of the irradiation is lost after approximately 1000 days. There is some discrepancy with the expected behaviour, because $V_{F D}$ becomes higher than $400 \mathrm{~V}$ and $500 \mathrm{~V}$ only after about 130 and 320 days of annealing, while the collected charge has already reduced. As expected, the signal measured at $600 \mathrm{~V}$ degrades at a lower rate. At this voltage, the signal is practically stable for about one year, then it degrades to a loss of $\sim 20 \%$ after 1000 days.

The n-in-p device shows instead a significant increase of the signal (up t0 30\%) during 100 days, followed by a slow degradation to retrieve the same level measured at the end of the irradiation after about 1000 days (Fig. 5). The signal is more than 20\% higher than its initial value during a time exceeding 300 equivalent days.

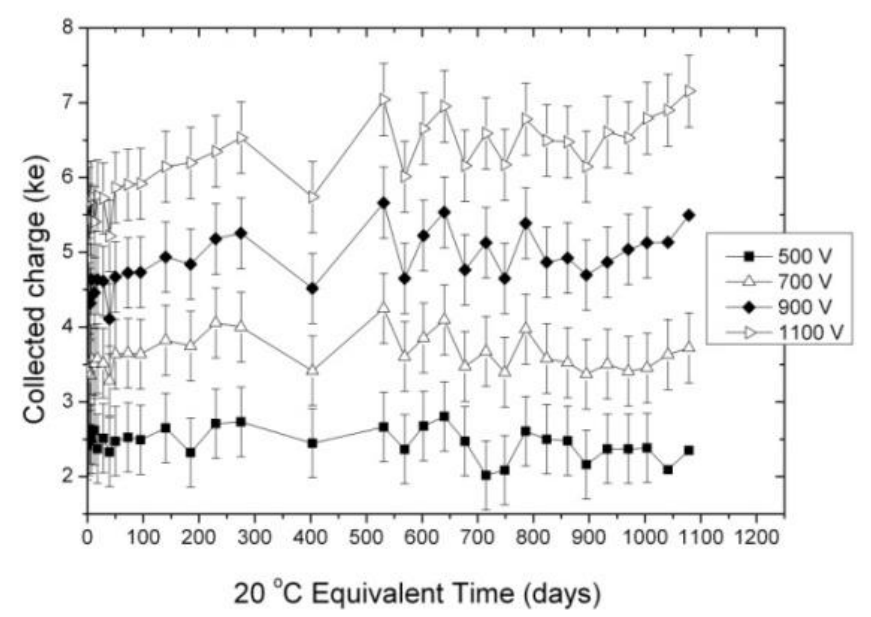

Figure 6: Signal as a function of the equivalent annealing time for a $n$-in-p detector irradiated with $26 \mathrm{MeV}$ protons to $1.5 \times 10^{16} \mathrm{n}_{\text {eq }} \mathrm{cm}^{-2}$. 


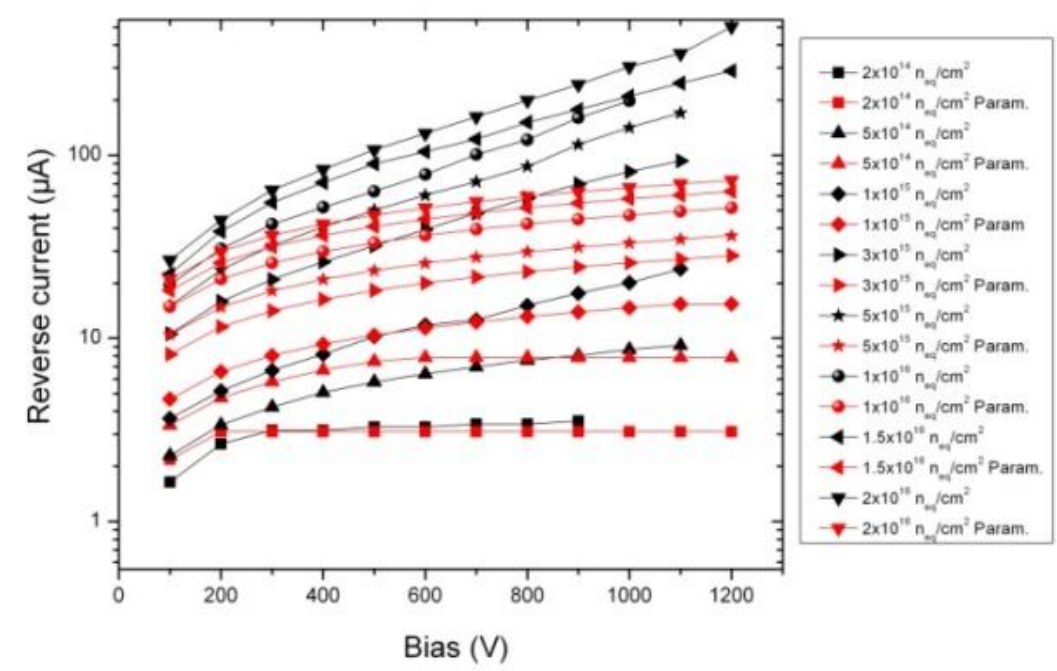

Figure 7: Expected (red) and measured reverse current as a function of the reverse bias voltage (I-V) for silicon detector irradiated to different doses.

After higher fluences of neutron irradiation the collected charge exhibits different annealing behaviours. Figure 6 shows the signal measured at four different reverse bias voltages (up to $1100 \mathrm{~V}$ ) for a microstrip sensor irradiated with $26 \mathrm{MeV}$ protons to $1.5 \times 10^{16} \mathrm{n}_{\mathrm{eq}} \mathrm{cm}^{-2}$ as a function of RT equivalent time. The increase of the measured signal is much slower than in the case of the sensors irradiated to lower doses, but it is still higher that the initial value for at least 1100 days at $20^{\circ} \mathrm{C}$.

\section{Changes of the reverse current with irradiation and annealing time}

Using the accepted parameterisation it is possible to calculate the reverse current at any irradiation dose and temperature. This is based on measurements performed up to fluences of about $1 \times 10^{15} \mathrm{n}_{\mathrm{eq}} \mathrm{cm}^{-2}$. But at higher hadron doses, the reverse current does not follow the expected behaviour. Figure 7 shows the comparison of the calculated and measured reverse current as a function of the reverse bias voltage for silicon detectors irradiated to different doses. At the lower fluences the difference is relatively small, with the exception of the points above $800 \mathrm{~V}$ for the detector irradiated to $1 \times 10^{15} \mathrm{n}_{\mathrm{eq}} \mathrm{cm}^{-2}$. At larger doses the measured currents are considerably higher than the calculated ones. This could be due to the same charge multiplication mechanism that is assumed to enhance the signal after irradiation.

Also the annealing of the reverse current with time after irradiation does not follow the parameterisation used to fit the data at lower doses. Because the charge multiplication effect could depend on various parameters of the segmented implants and on the junction profile, a general parameterisation of the reverse current at higher doses for the irradiated silicon microstrip detectors might not be possible and direct measurements with a given type of sensors could be required. Nonetheless the general trend of a relatively fast decrease of the current with time after irradiation is verified. Figure 7 shows the annealing behaviour of the reverse current for detectors irradiated with neutrons to $1 \times 10^{15} \mathrm{n}_{\mathrm{eq}} \mathrm{cm}^{-2}$ and with $26 \mathrm{MeV}$ protons to $1.5 \times 10^{16} \mathrm{n}_{\text {eq }}$ 
$\mathrm{cm}^{-2}$ A significant decrease of $I_{R}$ takes place already in the first $50 t_{\text {eq }}$ days, with a reduction of about $40 \%$. The $I_{R}$ keeps than decreasing with time at a slower rate.
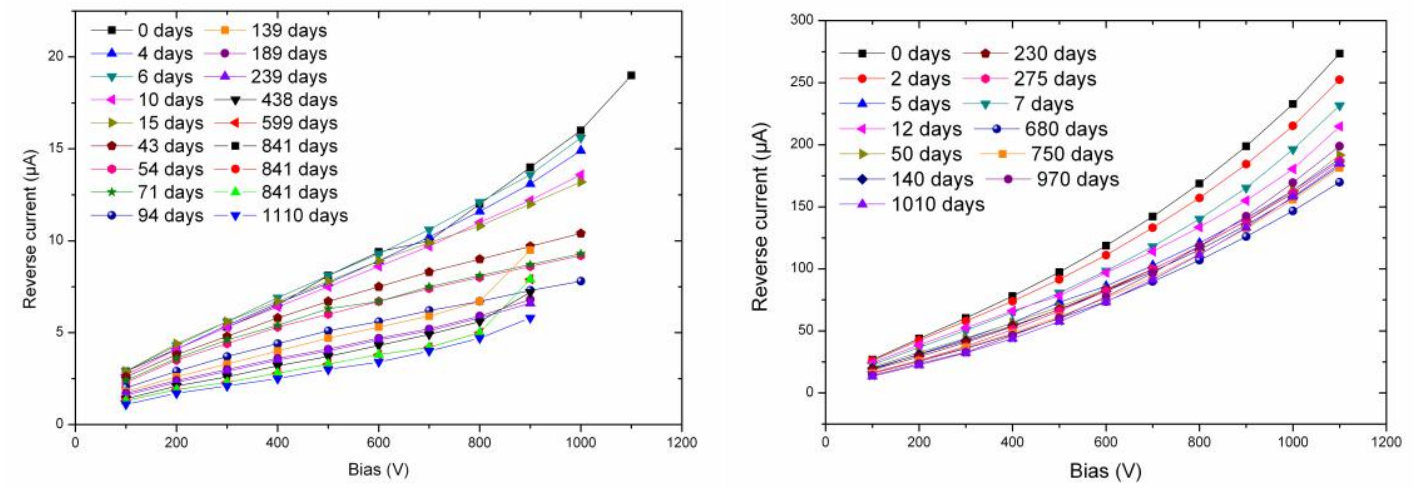

Figure 8: Annealing of the reverse current for detectors irradiated with neutrons to $1 \times 1^{15} n_{\text {eq }} \mathrm{cm}^{-2}$ (left) and with protons to $1.5 \times 1^{16} n_{e q} \mathrm{~cm}^{-2}$ ). The various annealing times are in $20^{\circ} \mathrm{C}$ equivalent days (the study has been accelerated at 60 and $80^{\circ} \mathrm{C}$ ).

\section{Implications for the operation scenarios in future super-colliders}

We attempt here to give general recommendations for operating high irradiated silicon detectors for high energy physics applications. Issues like the signal over noise are not considered, although essential to assess the efficiency of the detectors, because of the dependence of the noise on the particular geometry. Figure 9 shows the degradation of the signal with hadron fluence at $500 \mathrm{~V}$ and $900 \mathrm{~V}$.

These plots can be used to read the expected signal after the dose of interest and to evaluate the $\mathrm{S} / \mathrm{N}$ for a specific electronics system. Remarkably, a signal of $\sim 5000$ electrons is still measured after $2 \times 10^{16} \mathrm{n}_{\mathrm{eq}} \mathrm{cm}^{-2}$, sufficient to guarantee a tracking efficiency close to $100 \%$ with an electron noise charge of 500. The data refer to the signal before annealing: they are therefore the minimum collected charge after every dose. The measurements have been taken at operation temperatures between $-15 /-25{ }^{\circ} \mathrm{C}$. After the highest doses, $-25{ }^{\circ} \mathrm{C}$ is required for long term stable operation (this requirement can be relaxed for lower doses, where for example $-15^{\circ} \mathrm{C}$ is sufficient for operating silicon sensors irradiated to $1 \times 1015 \mathrm{neq} \mathrm{cm}-2$ ). There is the possibility of increasing the signal by as much as $30 \%$ by means of controlled annealing outside of physics data taking periods. The most significant improvement is found with the sensor irradiated to $1 \times 10^{15} \mathrm{n}_{\mathrm{eq}} \mathrm{cm}^{-2}$ and the highly irradiated sensor shows a signal that does not degrade with time after irradiation. Due to the dependence of the signal recovery on the fluence further measurements are required to experimentally assess the improvement of the collected charge with annealing time after various doses. An undisputable advantage of the annealing is the reduction of the current of the devices with time after irradiation. Namely, it can be concluded that an annealing time of about 50 days at room temperature can reduce the reverse current by $40 \%$ for both the fluences shown (Fig. 8). In the absence of an accurate model for describing the current and its annealing for severely irradiated silicon detectors, data from direct measurements should be employed. Table 1 shows an example of the power dissipated per $\mathrm{cm}^{2}$ by silicon detectors biased at $500 \mathrm{~V}$ and $1000 \mathrm{~V}$ and operated at $-25^{\circ} \mathrm{C}$ after various fluences of hadron irradiation. 

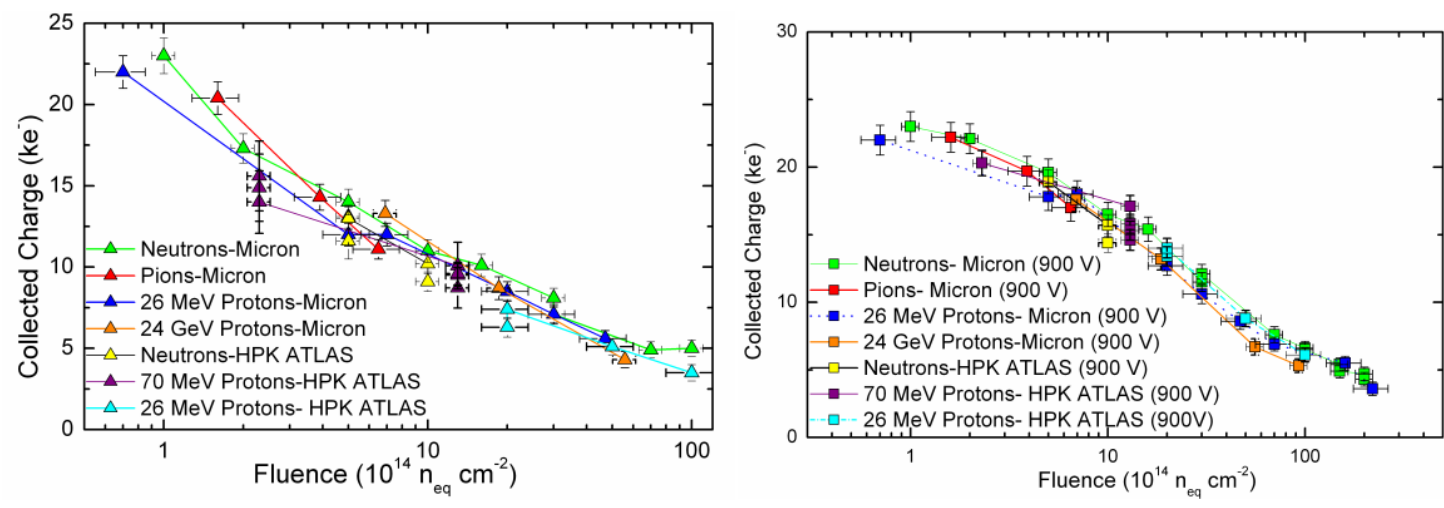

Figure 9. Degradation of the signal of n-in-p $300 \mu$ m thick sensors irradiated with different hadrons as a function of fluence at $500 \mathrm{~V}$ (left) and $900 \mathrm{~V}$ (right).

\section{Conclusions}

Planar silicon sensors exhibit higher than expected charge collection efficiency after irradiation, adequate for efficient tracking in all the layers of the upgraded experiments at the sLHC, provided that adequate cooling and power can be routed to the devices. Operating temperature of $-25^{\circ} \mathrm{C}$ and bias voltages of up to $1000 \mathrm{~V}$ are required for the innermost pixel layers, where hadron fluences of $2 \times 10^{16} \mathrm{n}_{\mathrm{eq}} \mathrm{cm}^{-2}$ can be expected. Although sub-zero temperatures are required during operations for preventing the thermal runaway of the reverse current and the consequent failure of the detectors, a noticeable improvement of the performance can be achieved by controlled annealing of the silicon sensors (e.g. at room temperature out of operation time).

It has also been shown that the accepted parameterisation of the changes of the electrical performance of the silicon sensors after hadron irradiation fails at high doses (above $1 \times 10^{15} \mathrm{n}_{\mathrm{eq}}$ $\mathrm{cm}^{-2}$ ). Above this dose the direct measurement of the signal and the reverse current should be used for anticipating the performance of the detector systems. Only the understanding of the mechanism responsible for the enhanced signal and increased reverse current in the highly dosed detectors (charge multiplication) will provide the model for describing the devices and accurately predict their performance without recurring to direct measurements.

\begin{tabular}{|c|c|c|c|c|c|}
\hline \multirow[t]{2}{*}{$\begin{array}{c}\text { Fluence } \\
\left(10^{15} n_{e q} \mathrm{~cm}^{-2}\right)\end{array}$} & \multicolumn{2}{|c|}{$\begin{array}{c}\text { Power }\left(\mathrm{m} \mathrm{W} / \mathrm{cm}^{2}\right) \text { at }-25^{\circ} \mathrm{C} \\
\text { (no annealing) }\end{array}$} & \multicolumn{2}{|c|}{$\begin{array}{c}\text { Power }\left(\mathrm{m} \mathrm{W} / \mathrm{cm}^{2}\right) \text { at }-25^{\circ} \mathrm{C} \\
\text { (45d RT annealing) }\end{array}$} & \multirow{2}{*}{$\begin{array}{c}\begin{array}{c}\text { Signal } \\
\text { (ke) }\end{array} \\
1000 \mathrm{~V}\end{array}$} \\
\hline & $500 \mathrm{~V}$ & $1000 \mathrm{~V}$ & $500 \mathrm{~V}$ & $1000 \mathrm{~V}$ & \\
\hline 5 & 24 & 65 & 14.4 & 39 & $>10$ \\
\hline 10 & 30.5 & 100 & 18.3 & 60 & $>7$ \\
\hline 15 & 46.5 & 115 & 27.9 & 69 & $>6$ \\
\hline 20 & 53.5 & 150 & 32.1 & 90 & $>4$ \\
\hline
\end{tabular}

Table 1: Power dissipation of silicon detectors biased at $500 \mathrm{~V}$ and $1000 \mathrm{~V}$ and operated at $-25^{\circ} \mathrm{C}$ after severe fluences of hadron irradiation.

\section{Acknowledgements}

The research here presented was performed within the framework of the RD50 collaboration. 
The authors are grateful to the colleagues at the CERN-PS irradiation facility, the JSI of Ljubljana and the Cyclotron at Karlsruhe with special thanks to M. Glaser, V. Cindro and A. Dierlamm for the irradiation of our devices.

\section{References}

[1] O. Bruhning et al., LHC Project Report 626.

[2] T. Dubbs et al.. Nucl. Instr. and Meth. A 383 (1996), p. 174.

[3] G. Casse, P.P. Allport, T.J.V. Bowcock, A. Greenall, M. Hanlon, J.N. Jackson, "First results on the charge collection properties of segmented detectors made with p-type bulk silicon", Nuclear Instruments and Methods in Physics Research vol. 487/3 (Jul. 2002) 465-470.

[4] G. Casse, P.P. Allport, S. Marti y Garcia, M Lozano, P.R. Turner, "Performances of miniature microstrip detectors made on oxygen enriched p-type substrates after very high proton irradiation", Nuclear Instruments and Methods in Physics Research A, vol. 535/1-2, (2004)362-365.

[5] I. Mandic, V. Cindro, G. Kramberger and M. Mikuž, "Observation of full charge collection efficiency in $\mathrm{n}+\mathrm{p}$ strip detectors irradiated up to $3 \times 10^{15} \mathrm{n} / \mathrm{cm}^{2 n}$, presented at $7^{\text {th }}$ International Conference on Radiation Effects on Semiconductor Materials Detectors and Devices, to be published in Nucl. Instrum. Meth. A.

[6] M.K. Petterson, R.F. Hurley, K. Arya, C. Betancourt, M. Bruzzi, B. Colby, M. Gerling, C. Meyer, et al., "Determination of the charge collection efficiency in neutron irradiated silicon detectors", IEEE Nuclear Science Symposium Conference Record, Oct. 26 2007-Nov. 3 2007 Vol. 2, pp 1329-1331.

[7] The ATLAS Collaboration, Inner Detector TDR, CERN LHCC 9716,9717 (1997)

[8] The CMS Collaboration, CERN/LHCC 98-6, (1998).

[9] G. Lindström et al., (The RD48 Collaboration). Nucl. Instr. and Meth A 466 (2001), p. 308.

[10] G. Kramberger, M. Batič, V. Cindro, I. Mandic, M. Mikuz and M. Zavrtanik, " Annealing studies of effective trapping times in silicon detectors", NIM A 571, Issue 3, 2007, 608-611.

[11] G. Casse, A. Affolder, P.P. Allport and M. Wormald, "Evaluation of floating zone and epitaxial planar silicon detectors with different substrate thickness after irradiation up to $2 \times 10^{16} \mathrm{n}_{\mathrm{eq}} \mathrm{cm}^{-2 "}$, accepted for publications in IEEE Trans. Nucl. Sci.

[12] G. Casse, A. Affolder, P.P. Allport and M. Wormald, "Enhanced efficiency of segmented silicon detectors of different thicknesses after hadron irradiations up to $2 \times 10^{16} \mathrm{n}_{\mathrm{eq}} \mathrm{cm}^{2 "}$, presented at the 11th ESSD, Wildbat Kreuth, 7-11 June 2009, accepted for publication in Nucl. Instr. and Meth $A$. 